Stefan de Haan

\title{
NEMS-emerging products and applications of nano-electromechanical systems
}

ABSTRACT. In this article we investigate points of contact between micro-electromechanical systems (MEMS) and nanotechnology. Several examples of companies harnessing nanoelectromechanical systems (NEMS) are presented, highlighting the fields in which a downscaling of MEMS will become commercially important. Although major MEMS companies do not feel concerned with nanotechnology, NEMS are likely to have an impact on mass markets in the fields of storage, sensing, radio frequency (RF) MEMS, and systems based on carbon nanotubes (CNTs).

Nanotechnology Perceptions 2 (2006) 267-275

Nonsubscribers: purchase individual article 